\title{
Guinea Pig Leukemia
}

National Cancer Institute

\section{Source}

National Cancer Institute. Guinea Pig Leukemia. NCI Thesaurus. Code C134564.

Leukemia that occurs in a guinea pig. 